$\$=-1$

\title{
Small and Medium Enterprises, Central Business District (CBD) for Accelerating of Regional Development
}

\author{
Nyoman Sudapet ${ }^{1}$, Agus Sukoco ${ }^{1}$, Muhammad Ikhsan Setiawan ${ }^{2 *}$, Paisal Halim ${ }^{3}$, Syamsiah Badruddin ${ }^{3}$, Tuswoyo ${ }^{3}$, \\ Ahmad Hidayat ${ }^{3}$, Darmawan Napitupulu ${ }^{4}$, Dahlan Abdullah ${ }^{5}$, A. Saleh A. ${ }^{6}$ \\ ${ }^{I}$ Department of Management, Narotama University, Surabaya, Indonesia \\ ${ }^{2}$ Department of Civil Engineering, Narotama University, Surabaya, Indonesia \\ ${ }^{3}$ Institut Ilmu Sosial dan Manajemen STIAMI, Jakarta, Indonesia \\ ${ }^{4}$ Research Center for Quality System and Testing Technology, Lembaga Ilmu Pengetahuan Indonesia, Jakarta, Indonesia \\ ${ }_{5}^{5}$ Department of Informatics, Universitas Malikussaleh, Aceh Utara, Indonesia \\ ${ }^{6}$ Financial Mathematics Research Group, AHMAR Institute, Indonesia \\ *Corresponding author E-mail: ikhsan.setiawan@narotama.ac.id
}

\begin{abstract}
Madura is small island, East Java Province, Indonesia, with the Surabaya - Madura (Suramadu) Bridge $5.7 \mathrm{~km}$ length. It is the largest bridges In Indonesia, connected 2 (two) island, Java and Madura. In Suramadu area will be build landed house and apartments, residential, central of business, central of tourism. In Suramadu area, especially in Surabaya side will be built by some interesting landed house and apartments, residential, central of business, central of tourism, combining with recreation area. The Government seeks to attract the private sector to cooperate in the development and investment in landed house and apartments, residential, central of business, central of tourism, through the approach of government and private cooperation. Law number 22/1999 and Law number 34/2004 on regional autonomy have improved the performance of local governments, in particular through the policy of increasing local revenues through cooperation with private parties. Investment must be injected in Suramadu area by investors is IDR 18,410,577,670,000.00, it would be very interesting.
\end{abstract}

Keywords: Digital SME's; Market Capitalization; Regional Center; Regional Economic

\section{Introduction}

Indonesia ranks high on investment by the Economist Corporate Network Asia Business Outlook Survey 2014, and top-ranked investment prospects by UNCTAD 2013-2015, Boston Consulting Group says the population of medium to high class increasing in Indonesia, reaching 74 million people (2013) and 141 million (2020), this is what causes an increase in domestic investment, especially in the investment of landed house and apartment ownership. BKPM (Indonesia Investment Board) released information on increasing investment especially in landed house and apartment ownership, residential, central of business, central of tourism which has increased significantly since 2010, 2014 has reached Rp.25.66 trillion. Increasing investment especially in landed house and apartment ownership, residential, central of business, central of tourism, has attracted government interest. The Government seeks to attract the private sector to cooperate in the development and investment in landed house and apartments, residential, central of business, central of tourism, through the approach of government and private cooperation. Law number 22/1999 and Law number $34 / 2004$ on regional autonomy have improved the performance of local governments, in particular through the policy of increasing local revenues through cooperation with private parties (BI, 2014; Economist, 2014; Home Affairs, 2013; Interior, 2010; Exchange, 2013; Landscape, 2015; UNCTAD, 2013; Consulting Group, 2013; Bappenas, 2017).
The development and investment in landed house and apartments, residential, central of business, central of tourism in some regions, become local government needed, because of total APBD (Local Government Budgeting) 2013 deficit IDR 54,217 trillion. So the development and investment will help local government budgeting (APBD). In 2010 total assets of government land IDR 558,456 trillion, total assets of government building Rp228.343 trillion, it is can be useful. Public-private partnerships become the answer of budgeting problem in some regions. best practices is PT Pembangunan Jaya Ancol Tbk Jakarta owned by provincial DKI Jakarta government $(72 \%)$, share of PT Pembangunan Jaya (18.01\%) and share of public (9.99\%), manage land area of 500 ha with recreation area of 200 ha. It's impact on government budgeting (APBD) in 2013 of DKI Jakarta Provincial Government, non-tax revenue and retribution IDR 3,252 trillion, income IDR 26,670.45 trillion, non-tax receipts and levies $12.19 \%$ including profit sharing of PT Pembangunan Jaya Ancol Tbk (public-private partnership) (Bappenas, 2017; Santosa, 2013; Novak, 2017; King, 2017; Ito, 2016; Belza, 2017; Ellen \& Williamson, 2017; Tarun, Ubeja, \& Chatterjee, 2017; Melodi \& Prawlall, 2017).

Government Law number 6/2006 on the management of territory assets, states that Assets may be utilized by business entities, in accordance with concessions, assets built by business entities for the benefit of the government, then operated by the Business Entity. Management of assets can be lease, leasing, joint use, build operate transfer (BOT) and build transfer order (BTO). Government Law number 50/2007 on Implementation of Regional Cooperation, states that cooperation between local government and 
business entities, must be approved by the Legislative Council, impact on revenue increased, the use of local government assets (Melodi \& Prawlall, 2017; Daniela \& Paiva, 2017; Okamoto, Yatsuhashi \& Mizutani, 2017; Rim \& Hussien, 2017; Lucia-Palacios, Perez-Lopez \& Polo-Redondo, 2017; Rina \& Sangodoyin, 2017; Hu, Fox, \& Qian, 2017; Janoschka \& Arreortua, 2017; Anthony, 2017; Wegmann \& Jiao, 2017; Bryant, Gillian, Halina, Giles, \& Patricia, 2017; Peczek, Justyna, Peczek, Martyniuk, 2017; Korkmaz, \& Kasin, 2017).

\section{Experimental Details}

This research using qualitative research method, with case studies analysis, that analyze the interrelations of events or conditions in specific context, can be qualitative or multiple information sources. Research sampling is BPWS area (Board of Development Suramadu Bridge Area). Started with secondary data, then using primary data from direct interview of stakeholders who will develop Suramadu area in Surabaya side. last, feasibility analysis of central business and central tourism, including analysis of Net Present Value (NPV), Payback Period and Internal Rate of Return (IRR) (Nguyen, Tran, Vu, \& Luu, 2017; Knaap, 2017; Cox, Bassi, Kolling, Procter, Flanders, Tamers, \& Araujo, 2017; Lai, Zheng, Choy, \& Wang, 2017; Fan, Wu, \& Yang, 2017; Danilina \& Chebotarev, 2017; Li \& Liu, 2017; Wetzstein, 2017; Cerezo, Sokol, Alkhaled, Reinhart, 2017).

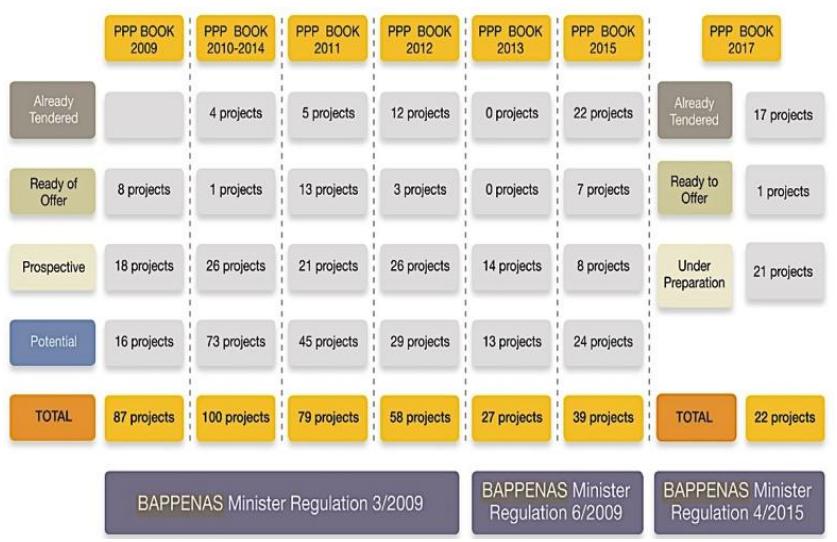

Fig. 1: PPP in Indonesia (Bappenas, 2017)

Net Present Value (NPV) is total revenue obtained during the project, reduced by the total cost and calculated based on the present value, with an interest rate. NPV calculated by discounting current annual costs and revenue, obtained the difference of these two amounts. This is model as follows: (Hu, Fox, \& Qian, 2017; Janoschka \& Arreortua, 2017; Anthony, 2017; Wegmann \& Jiao, 2017; Bryant, Gillian, Halina, Giles, \& Patricia, 2017; Peczek, Justyna, Peczek, Martyniuk, 2017; Korkmaz, \& Kasin, 2017; Nguyen, Tran, Vu, \& Luu, 2017; Knaap, 2017; Cox, Bassi, Kolling, Procter, Flanders, Tamers, \& Araujo, 2017; Lai, Zheng, Choy, \& Wang, 2017; Fan, Wu, \& Yang, 2017; Danilina \& Chebotarev, 2017; Li \& Liu, 2017; Wetzstein, 2017; Cerezo, Sokol, Alkhaled, Reinhart, 2017; Li, Wang, \& Chang, 2017; Lee, Kim, Parrott, Giddings, \& Robinson, 2017; Ghavidelfar, Shamseldin, \& Melville, 2017)

NPV = PV (pendapatan $\mathrm{x}$ faktor diskonto) - PV (biaya $\mathrm{x}$ faktor diskonto)

a. If the NPV is negative means that the construction project did not generate a profit in the economic life of the plan.

b. If the NPV is positive means that the construction project can be implemented for making a profit on the economic life of the plan. c. If the NPV equal to zero means that the construction project can be implemented but do not make a profit on the economic life of the plan.

The payback period is the time period required to return the investment made by the total present value of the cash flows generated. Internal Rate of Return (IRR) expressed as an interest rate or discount rate where the present value of the benefit is equal to the present value of the costs incurred on interest rates generated. In other words IRR is the discount rate or where NPV $=0$ or BCR $=$ 1.0. This method is formulated as follows: (Widjaya \& Tanuwidjaya, 2017; Nuraini, Prifiharni, Priyotomo, Sundjono, \& Gunawan, 2017; Ayuningtyas, 2017; Pribadi, 2015; Putra, Riyanto, Harsoyo, \& Kistijantoro, 2015; Yuanita, Rini, Heriawan, 2011; Huang, Ging, \& Dai, 2011).

$$
I R R=D f P+\frac{N P V P \times(D f N-D f P)}{N P V p-N P V n}
$$

Where,

$\mathrm{IRR}=$ Return on average

$\mathrm{DFP}=\mathrm{Df}$ is used to generate Net Present Value Positive

$\mathrm{DFN}=\mathrm{Df}$ is used to generate Net Present Value Negative

NPV $p=N P V$ at a discount a positive average

$\mathrm{NPV} n=\mathrm{NPV}$ at an average discount of negative

If IRR> applicable interest rate is feasible Prospect.

\section{Results and Discussion}

Madura are small island, East Java Province, Indonesia, with the Surabaya - Madura (Suramadu) Bridge $5.7 \mathrm{~km}$ length. It is the largest bridges In Indonesia, connected 2 (two) island, Java and Madura. In Suramadu area will be build landed house and apartments, residential, central of business, central of tourism.

In Suramadu area, especially in Surabaya side will be built by some interesting landed house and apartments, residential, central of business, central of tourism, combining with recreation area.

This region had four districts. 4 districts where it had kind of building as follows:

1. Area Development 1 , total of 6 buildings consisting of: (a) Office Tower by 2 buildings; (b) Commercial and Urban Housing as many as two buildings; (c) Urban Housing as many as one building; (d) The apartments in 1 building.

2. Area Development 2, total of 2 buildings consisting of: (a) Commercial much as 2 buildings:

3. Area Development 3, total of 4 buildings consisting of: (a) Urban Housing as many as 3 buildings; (b) Community center as much as 1 building.

4. Area Development 4 , total of 11 buildings comprising: (a) Urban Housing as much as 6 building; (b) Commercial much as 5 buildings.

Based on the plan that made the planning area into 4 Area Development, amount of landed house and apartment investment, residential investment, central of business investment, central of tourism investment would vary from one Area Development to another Area Development due to differences in value. Difference occurred because the location, differences in the selling price. In this regard, it was based on market prices estimated price could be as follows:

1. Area Development 1, there were 6 buildings had an estimated price of land each of the buildings is as follows:

Table 1: Area development 1 with six buildings analysis (in IDR)

\begin{tabular}{lllrr}
\hline Block & \multicolumn{1}{c}{ Building } & $\begin{array}{c}\text { Area } \\
\left(\mathbf{m}^{2}\right)\end{array}$ & Cost $/ \mathbf{m}^{\mathbf{2}}$ & \multicolumn{1}{c}{ Total Cost } \\
\hline A1 & Office Tower & 45,423 & 750,000 & $34,067,250,000$ \\
A2 & Office Tower & 41,385 & $4,500,000$ & $186,232,500,000$ \\
B1 & Commercial and & 66,610 & $2,625,000$ & $174,851,250,000$ \\
& Urban Housing & & & \\
B2 & Commercial and & 30,850 & $1,500,000$ & $46,275,000,000$
\end{tabular}




\begin{tabular}{|c|c|c|c|c|}
\hline & Urban Housing & & & \\
\hline B3 & Urban Housing & 48,348 & $1,500,000$ & $72,522,000,000$ \\
\hline $\mathrm{C} 3$ & $\begin{array}{l}\text { Apartment and } \\
\text { Commercial }\end{array}$ & 42,500 & 750,000 & $31,875,000,000$ \\
\hline
\end{tabular}

2. Area Development 2, there were 2 buildings had a land price estimate of each building are as follows:

Table 2: Area development 2 with 2 buildings (in IDR)

\begin{tabular}{lcccc}
\hline Block & Building & $\begin{array}{c}\text { Area } \\
\left(\mathbf{( m}^{2}\right)\end{array}$ & Cost $/ \mathbf{m}^{\mathbf{2}}$ & Total Cost \\
\hline C1 & Commercial & 45,005 & $2,125,000$ & $89,260,625,000$ \\
C2 & Commercial & 43,250 & 500,000 & $21,625,000,000$ \\
\hline 3. & Area Development 3, there were & 4 buildings had an approxi- \\
\multicolumn{5}{c}{ mate price of each Building land, are as follows: }
\end{tabular}

Table 3: Area development 3 with four buildings (in IDR)

\begin{tabular}{lllrr}
\hline Block & \multicolumn{1}{c}{ Building } & $\begin{array}{c}\text { Area } \\
\left(\mathbf{m}^{2}\right)\end{array}$ & \multicolumn{1}{c}{ Cost $/ \mathbf{m}^{\mathbf{2}}$} & \multicolumn{1}{c}{ Total Cost } \\
\hline D1 & Urban Housing & 39,404 & $2,125,000$ & $83,733,500,000$ \\
D2 & Urban Housing & 15,951 & 750,000 & $11,963,250,000$ \\
D3 & Urban Housing & 22,910 & $4,500,000$ & $103,095,000,000$ \\
E1 & Commercial & 18,165 & $2,125,000$ & $38,600,625,000$ \\
& Center & & & \\
\hline
\end{tabular}

4. Area Development 4, there were 11 buildings had a land price estimate each Building are as follows:

Table 4: Area development 4 with 11 buildings (in IDR)

\begin{tabular}{llrlc}
\hline Block & Building & \multicolumn{1}{c}{ Area $\left(\mathbf{m}^{2}\right)$} & Cost $/ \mathbf{m}^{2}$ & Total Cost \\
\hline D4 & Urban Housing & $11,147.59$ & $2,500,000$ & $27,868,975,000$ \\
D5 & Urban Housing & $9,841.75$ & $1,500,000$ & $14,762,625,000$ \\
D6 & Urban Housing & $8,225.99$ & $3,500,000$ & $28,790,965,000$ \\
D7 & Urban Housing & $8,543.62$ & $2,500,000$ & $21,359,050,000$ \\
D8 & Urban Housing & $17,350.14$ & $2,500,000$ & $43,375,350,000$ \\
D9 & Urban Housing & $22,830.63$ & $2,500,000$ & $57,076,535,000$ \\
F1 & Commercial & $12,065.58$ & $4,500,000$ & $54,295,110,000$ \\
F2 & Commercial & $11,819.35$ & $3,500,000$ & $41,367,725,000$ \\
F3 & Commercial & $21,573.07$ & $1,500,000$ & $32,359,605,000$ \\
F4 & Commercial & $17,120.96$ & $1,500,000$ & $25,681,440,000$ \\
F5 & Commercial & $10,968.71$ & $2,500,000$ & $27,396,775,000$ \\
\hline
\end{tabular}

Area Development cost of construction for buildings is different.

Cost of building construction varies due to differences in function, design and architecture. Estimated cost of building in Area Development based on estimated cost, as follows:

1. Area Development 1, there were 6 building had an estimated cost of physical development are as follows:

Table 5: Area development 1 with 6 building analysis (in IDR)

\begin{tabular}{|c|c|c|c|c|}
\hline Block & Building & $\begin{array}{l}\text { Area } \\
\left(\mathbf{m}^{2}\right)\end{array}$ & $\operatorname{Cost} / \mathrm{m}^{2}$ & Total Cost \\
\hline A1 & Office Tower & 303,600 & $6,000,000$ & $1,821,600,000,000$ \\
\hline $\mathrm{A} 2$ & Office Tower & 292,100 & $6,000,000$ & $1,752,600,000,000$ \\
\hline B1 & $\begin{array}{l}\text { Commercial } \\
\text { and Urban } \\
\text { Housing }\end{array}$ & 328,300 & $7,000,000$ & $2,298,100,000,000$ \\
\hline B2 & $\begin{array}{l}\text { Commercial } \\
\text { and Urban } \\
\text { Housing }\end{array}$ & 136,400 & $7,000,000$ & $954,800,000,000$ \\
\hline B3 & $\begin{array}{l}\text { Urban Hous- } \\
\text { ing }\end{array}$ & 259,350 & $4,500,000$ & $1,167,075,000,000$ \\
\hline $\mathrm{C} 3$ & $\begin{array}{l}\text { Apartment and } \\
\text { Commercial }\end{array}$ & 158,000 & $7,500,000$ & $1,185,000,000,000$ \\
\hline
\end{tabular}

2. Area Development 2, there were 2 buildings had physical construction cost estimates are as follows:

Table 6: Area development 2 with 2 building analysis (in IDR)

\begin{tabular}{llllc}
\hline Block & Building & Area $\left(\mathbf{m}^{2}\right)$ & Cost $/ \mathbf{m}^{2}$ & Total Cost \\
\hline C1 & Commercial & 284,200 & $8,000,000$ & $2,273,600,000$ \\
C2 & Commercial & 182,634 & $8,000,000$ & $1,461,072,000$ \\
\hline
\end{tabular}

3. Area Development 3, there were 4 buildings had physical construction cost estimates are as follows:

Table 7: Area development 3 with 4 building analysis (in IDR)

\begin{tabular}{llllc}
\hline Block & \multicolumn{1}{c}{ Building } & \multicolumn{1}{c}{ Area $\left(\mathbf{m}^{2}\right)$} & \multicolumn{1}{c}{ Cost $/ \mathbf{m}^{2}$} & \multicolumn{1}{c}{ Total Cost } \\
\hline D1 & Urban Housing & $185,072.49$ & $4,500,000$ & $832,826,705,000$ \\
D2 & Urban Housing & 86,896 & $4,500,000$ & $391,032,000,000$
\end{tabular}

\begin{tabular}{lllll} 
D3 & Urban Housing & $76,816.5$ & $4,500,000$ & $345,674,250,000$ \\
E1 & Commercial & 31,761 & $8,000,000$ & $254,088,000,000$ \\
& Center & & & \\
\hline
\end{tabular}

4. Area Development 4 , there were 11 buildings had physical construction cost estimates are as follows:

Table 8: Area development 4 with 11 building (in IDR)

\begin{tabular}{|c|c|c|c|c|}
\hline Block & Building & Area $\left(\mathrm{m}^{2}\right)$ & Cost $/ \mathrm{m}^{2}$ & Total Cost \\
\hline D4 & $\begin{array}{l}\text { Urban Hous- } \\
\text { ing }\end{array}$ & $19,992.29$ & $4,500,000$ & $89,865,305,000$ \\
\hline D5 & $\begin{array}{l}\text { Urban Hous- } \\
\text { ing }\end{array}$ & $19,241.58$ & $4,500,000$ & $86,587,110,000$ \\
\hline D6 & $\begin{array}{l}\text { Urban Hous- } \\
\text { ing }\end{array}$ & $10,368.28$ & $4,500,000$ & $46,657,260,000$ \\
\hline D7 & $\begin{array}{l}\text { Urban Hous- } \\
\text { ing }\end{array}$ & $17,920.00$ & $4,500,000$ & $80,640,000,000$ \\
\hline D8 & $\begin{array}{l}\text { Urban Hous- } \\
\text { ing }\end{array}$ & $35,915.00$ & $4,500,000$ & $161,617,500,000$ \\
\hline D9 & $\begin{array}{l}\text { Urban Hous- } \\
\text { ing }\end{array}$ & $47,808.00$ & $4,500,000$ & $215,136,000,000$ \\
\hline F1 & Commercial & $14,416.26$ & $8,000,000$ & $115,330,080,000$ \\
\hline $\mathrm{F} 2$ & Commercial & $14,120.13$ & $8,000,000$ & $112,961,040,000$ \\
\hline F3 & Commercial & $31,921.00$ & $8,000,000$ & $255,368,000,000$ \\
\hline $\mathrm{F} 4$ & Commercial & $116,082.51 .00$ & $8,000,000$ & $928,660,080,000$ \\
\hline F5 & Commercial & $38,994.40$ & $8,000,000$ & $311,955,200,000$ \\
\hline
\end{tabular}

Based on the calculation cost of land and the construction of estimated investment value, that must be spent each building are as follows:

1. Area Development 1 , there were 6 building had an estimated value of the investment is as follows:

Table 9: Area development 1 with 6 building analysis (in IDR)

\begin{tabular}{|c|c|c|c|c|}
\hline $\begin{array}{c}\text { Bloc } \\
k\end{array}$ & Building & Area $\left(\mathrm{m}^{2}\right)$ & $\operatorname{Cost} / \mathrm{m}^{2}$ & Total Cost \\
\hline \multirow[t]{2}{*}{ A1 } & Office & $34,067,250,000$ & $1,821,600,000,0$ & $1,855,667,250,0$ \\
\hline & Tower & & 00 & 00 \\
\hline \multirow[t]{2}{*}{ A2 } & Office & $186,232,500,00$ & $1,752,600,000,0$ & $1,938,832,500,0$ \\
\hline & Tower & 0 & 00 & 00 \\
\hline \multirow[t]{2}{*}{ B1 } & Commer- & $174,851,250,00$ & $2,298,100,000,0$ & $2,472,951,250,0$ \\
\hline & $\begin{array}{l}\text { Urban } \\
\text { Housing }\end{array}$ & & & \\
\hline \multirow[t]{2}{*}{ B2 } & $\begin{array}{l}\text { Commer- } \\
\text { cial and } \\
\text { Urban }\end{array}$ & $46,275,000,000$ & $954,800,000,000$ & $\begin{array}{r}1,001,075,000,0 \\
00\end{array}$ \\
\hline & Housing & & & \\
\hline \multirow[t]{2}{*}{ B3 } & Urban & $72,522,000,000$ & $1,167,075,000,0$ & $1,239,597,000,0$ \\
\hline & Housing & & 00 & 00 \\
\hline \multirow[t]{3}{*}{$\mathrm{C} 3$} & Apartment & $31,875,000,000$ & $1,185,000,000,0$ & $1,216,875,000,0$ \\
\hline & $\begin{array}{l}\text { and Com- } \\
\text { mercial }\end{array}$ & & 00 & 00 \\
\hline & TOTAL & $\begin{array}{r}545,823,000,00 \\
0\end{array}$ & $\begin{array}{r}9,179,175,000,0 \\
00\end{array}$ & $\begin{array}{r}9,724,998,000,0 \\
00\end{array}$ \\
\hline
\end{tabular}

2. Area Development 2, there were 2 building had an estimated value of the investment is as follows:

Table 10: Area development 2 with 2 building analysis (in IDR)

\begin{tabular}{clrrr}
$\begin{array}{c}\text { Bloc } \\
\mathbf{k}\end{array}$ & Building & Area $\left(\mathbf{m}^{\mathbf{2}}\right)$ & Cost $/ \mathbf{m}^{\mathbf{2}}$ & \multicolumn{1}{c}{ Total Cost } \\
\hline $\mathrm{C} 1$ & $\begin{array}{l}\text { Commer- } \\
\text { cial }\end{array}$ & $89,260,625,000$ & $2,273,600,000,00$ & $2,362,860,625,00$ \\
& & 0 & 0 \\
$\mathrm{C} 2$ & $\begin{array}{l}\text { Commer- } \\
\text { cial }\end{array}$ & $21,625,000,000$ & $1,461,072,000,00$ & $1,482,697,000,00$ \\
& TOTAL & $\mathbf{1 1 0 , 8 8 5 , 6 2 5 , 0 0}$ & 0 & 0 \\
\hline & & $\mathbf{3 , 7 3 4 , 6 7 2 , 0 0 0 , 0 0}$ & $\mathbf{3 , 8 4 5 , 5 5 7 , 6 2 5 , 0 0}$ \\
& & & $\mathbf{0}$ & $\mathbf{0}$ \\
\hline
\end{tabular}

3. Area Development 3, there were 4 building had an estimated value of the investment is as follows:

Table 11: Area development 3 with 4 building analysis (in IDR)

\begin{tabular}{clrrr}
\hline $\begin{array}{c}\text { Bloc } \\
\text { k }\end{array}$ & Building & Area $\left(\mathbf{m}^{2}\right)$ & Cost $/ \mathbf{m}^{2}$ & Total Cost \\
\hline D1 & Urban & $83,733,500,0$ & $832,623,705,00$ & $916,357,205,00$ \\
& Housing & 00 & 0 & 0 \\
D2 & Urban & $11,963,250,0$ & $391,032,000,00$ & $402,995,250,00$ \\
& Housing & 00 & 0 & 0 \\
D3 & Urban & $103,095,000$, & $345,674,250,00$ & $448,769,250,00$ \\
& Housing & 000 & 0 & 0 \\
E1 & Commer- & $38,600,625,0$ & $254,088,000,00$ & $292,688,625,00$ \\
\hline
\end{tabular}




\begin{tabular}{lrrr}
$\begin{array}{lrr}\text { cial Cen- } \\
\text { ter }\end{array}$ & 00 & 0 & 0 \\
\hline TOTAL & $\mathbf{2 3 7 , 3 9 2 , 3 7 5 ,}$ & $\mathbf{1 , 8 3 2 , 4 1 7 , 9 5 5 ,}$ & $\mathbf{2 , 0 6 0 , 8 1 0 , 3 3 0 ,}$ \\
& $\mathbf{0 0 0}$ & $\mathbf{0 0 0}$ & $\mathbf{0 0 0}$ \\
\hline
\end{tabular}

4. Area Development 4, there were 11 building had an estimated value of the investment is as follows:

Table 12: Area development 4 with 11 building analysis (in IDR)

\begin{tabular}{|c|c|c|c|c|}
\hline $\begin{array}{c}\text { Bloc } \\
\mathbf{k}\end{array}$ & Building & $\operatorname{Area}\left(\mathbf{m}^{2}\right)$ & Cost $/ \mathrm{m}^{2}$ & Total Cost \\
\hline \multirow[t]{2}{*}{ D4 } & Urban & $27,868,975,0$ & $89,965,305,000$ & $117,834,280,00$ \\
\hline & Housing & 00 & & 0 \\
\hline \multirow[t]{2}{*}{ D5 } & Urban & $14,762,625,0$ & $86,587,110,000$ & $101,349,735,00$ \\
\hline & Housing & 00 & & 0 \\
\hline \multirow[t]{2}{*}{ D6 } & Urban & $28,790,965,0$ & $46,657,260,000$ & $75,448,225,000$ \\
\hline & Housing & 00 & & \\
\hline \multirow[t]{2}{*}{ D7 } & Urban & $21,359,050,0$ & $80,640,000,000$ & $101,999,050,00$ \\
\hline & Housing & 00 & & 0 \\
\hline \multirow[t]{2}{*}{ D8 } & Urban & $43,375,350,0$ & $161,617,500,00$ & $204,992,850,00$ \\
\hline & Housing & 00 & 0 & 0 \\
\hline \multirow[t]{2}{*}{ D9 } & Urban & $57,076,525,0$ & $215,136,000,00$ & $272,212,525,00$ \\
\hline & Housing & 00 & 0 & 0 \\
\hline \multirow[t]{2}{*}{ F1 } & Commer- & $54,295,110,0$ & $115,330,080,00$ & $169,625,190,00$ \\
\hline & cial & 00 & 0 & 0 \\
\hline \multirow[t]{2}{*}{$\mathrm{F} 2$} & Commer- & $41,367,725,0$ & $112,961,040,00$ & $154,328,765,00$ \\
\hline & cial & 00 & 0 & 0 \\
\hline \multirow[t]{2}{*}{ F3 } & Commer- & $32,359,605,0$ & $255,368,000,00$ & $287,727,605,00$ \\
\hline & cial & 00 & 0 & 0 \\
\hline \multirow[t]{2}{*}{ F4 } & Commer- & $25,681,440,0$ & $928,660,080,00$ & $954,341,520,00$ \\
\hline & cial & 00 & 0 & 0 \\
\hline \multirow[t]{3}{*}{ F5 } & Commer- & $27,396,775,0$ & $311,955,200,00$ & $339,251,975,00$ \\
\hline & cial & 00 & 0 & 0 \\
\hline & TOTAL & $\begin{array}{r}374,334,145, \\
000\end{array}$ & $\begin{array}{r}2,404,877,570 \\
000\end{array}$ & $\begin{array}{r}2,779,211,715 \\
000\end{array}$ \\
\hline
\end{tabular}

Based on the calculations of Area Development 1, Area Development 2, Area Development 3 and Area Development 4, total investment must be injected by investors is IDR $18,410,577,670,000.00$ detailed in each district as follows:

Table 13: Total investment of area development 1, area development 2, area development 3 and area development 4 (in IDR)

\begin{tabular}{|c|c|c|c|}
\hline Block & Total cost & N Present Value & Present Value \\
\hline 1 & $545,823,000,000$ & $9,179,175,000,000$ & $9,724,998,000,000$ \\
\hline 2 & $110,885,625,000$ & $3,734,672,000,000$ & $3,845,557,625,000$ \\
\hline 3 & $237,392,375,000$ & $1,832,417,955,000$ & $2,060,810,330,000$ \\
\hline 4 & $374,334,145,000$ & $2,404,877,570,000$ & $2,779,211,715,000$ \\
\hline TO- & $1,268,435,145,00$ & $17,142,142,525,00$ & $18,410,577,670,00$ \\
\hline TAL & 0 & 0 & 0 \\
\hline
\end{tabular}

\section{Conclusion}

Madura is small island, East Java Province, Indonesia, with the Surabaya - Madura (Suramadu) Bridge $5.7 \mathrm{~km}$ length. It is the largest bridges In Indonesia, connected 2 (two) island, Java and Madura.

In Suramadu area will be build landed house and apartments, residential, central of business, central of tourism. In Suramadu area, especially in Surabaya side will be built by some interesting landed house and apartments, residential, central of business, central of tourism, combining with recreation area.

The Government seeks to attract the private sector to cooperate in the development and investment in landed house and apartments, residential, central of business, central of tourism, through the approach of government and private cooperation.

Law number 22/1999 and Law number 34/2004 on regional autonomy have improved the performance of local governments, in particular through the policy of increasing local revenues through cooperation with private parties.

Investment must be injected in Suramadu area by investors is IDR $18,410,577,670,000$, it would be very interesting.

\section{Acknowledgment}

This paper is a part of a Kementerian Ristek DIKTI Research Grant titled "Model of Integration: Maritime and Tourism in Local Area, To Increase Economic Growth of East Indonesia" with Drs.Ec. I Nyoman Sudapet, MM, Agus Sukoco, ST, MM, Muhammad Ikhsan Setiawan, ST, MT as the researchers.

\section{References}

[1] A. T. Nguyen, T. Q. Tran, H. V. Vu \& D. Q. Luu. (2017). International J. of Urban Sustainable Development

[2] B. Indonesia. (2014). Perkembangan Properti Komersial-Triwulan II 2014. Jakarta: Indonesia

[3] B. Consulting Group. (2013). Asia's Next Big Opportunity: Indonesia's Rising Middle-Class and Affluent Consumers.

[4] B. Melodi \& T. Prawlall. (2017). South African Journal of Business Management 48, 3

[5] BAPPENAS. (2017).Public Private Partnerships, Infrastructure Projects Plan in Indonesia, Minister Of National Development Planning / Head Of National Development Planning Agency. Jakarta

[6] Belza, C. E. Miyawaki, P. Allen, D. K. King, D. X. Marquez, D. L. Jones, S. Janicek, D. Rosenberg \& D. R. Brown. (2017). Building Community: Stakeholder Perspectives on Walking in Malls and Other Venues." Journal of aging and physical activity

[7] Bryant, L. Gillian, T. K. Halina, E. S. H. Giles \& A. F. Patricia. (2017). Urban Forestry \& Urban Greening

[8] C. Cerezo, J. Sokol, S. AlKhaled, C. Reinhart, A. Al-Mumin \& A. Hajiah. (2017). Energy and Buildings 154

[9] Dhaniarti, A. Wulandari, \& M. I. Setiawan. (2017). J. Darussalam: J. Pend., Kom. dan Pem. Huk. Isl., 8, 2 (2017).

[10] E. I. Danilina \& V. E. Chebotarev. (2017). Theoretical and Empirical Researches in Urban Management 12, 4

[11] E. Knaap. (2017). Housing Policy Debate 27, 6

[12] F. Daniela \& D. Paiva. (2017). The International Review of Retail, Distribution and Consumer Research

[13] F. Y. Xu \& A. R. Chen. (2009). China Civ. Eng. J 42, 1

[14] G. Dai, W. Gong, X. Zhao \& X. Zhou. (2010). Static testing of pilebase post-grouting piles of the Suramadu bridge

[15] Hu, Z. Y. Fox \& J. Qian. (2017). Land Use Policy 69

[16] Ito. (2016). Medical mall founders' satisfaction and integrated management requirements, The International journal of health planning and management.

[17] J. Anthony. (2017). J. of Urban Planning and Development 143, 4

[18] J. Ellen \& J. Williamson. (2017). Architectural Design 87, 5

[19] J. M. Widjaya \& G. Tanuwidjaja. (2017). IOP Conference Series: Earth and Environmental Science, vol. 79, no. 1, p. 012025. IOP Publishing

[20] J. Suyono, Suhermin, A. Sukoco, \& M. I. Setiawan. (2017). J. Darussalam: J. Pend., Kom. dan Pem. Huk. Isl., 8, 2

[21] J. Santosa. (2013). Public Private Partnership in Indonesia and ASEAN-East Java Case Study, PT Sarana Multi Infrastruktur (Persero). Jakarta, Indonesia

[22] J. Wegmann \& J. Jiao. (2017). Land Use Policy 69

[23] K. A. Korkmaz, Kasim A. (2017). IOP Conference Series: Materials Science and Engineering, 245, 8.

[24] K. Tarun, S. Ubeja \& A. S. Chatterjee. (2017). Vision 21, 3

[25] L. Cox, A. Bassi, J. Kolling, A. Procter, N. Flanders, N. Tanners \& R. Araujo. (2017). Landscape and Urban Planning 167

[26] L. Lucia-Palacios, R. Pérez-López \& Y. Polo-Redondo. (2017). Journal of Strategic Marketing

[27] L. Nuraini, S. Prifiharni, G. Priyotomo, Sundjono \& H. Gunawan. (2017). AIP Conference Proceedings, vol. 1823, no. 1, p. 020101. AIP Publishing

[28] Landscape: Asia Business Outlook Survey 2015, The Economist Corporate Network (2015)

[29] M. Ayuningtyas. (2017). IOP Conference Series: Earth and Environmental Science, vol. 54, no. 1, p. 012078. IOP Publishing

[30] M. Janoschka \& L. S. Arreortua. (2017). Habitat International 70

[31] M. I. Setiawan, S. W. Mudjanarko, R. D. Nasihien, \& C. Hasyim. (2017). ADRI Inter. J. of Sci., Eng. and Tech., 1,

[32] M. Novak. (2017). J. of Urban Regeneration \& Renewal 10, 2

[33] M. of Home Affairs. (2013). Posture budget for Fiscal Year 2013. Jakarta, Indonesia

[34] M. of the Interior. (2010). Balance of budget 2010. Jakarta, Indonesia 
[35] M. Peczek, Justyna, G. Peczek \& O. Martyniuk. (2017). IOP Conference Series: Materials Science and Engineering, 245, 8, IOP Publishing.

[36] M. Rim \& H. A. Hussien. (2017). International Review for Spatial Planning and Sustainable Development 5, 3

[37] M. Rina \& O. Sangodoyin. (2017). The International Review of Retail, Distribution and Consumer Research

[38] N. Sudapet, A. Sukoco, \& M. I. Setiawan. (2017). J. Darussalam: J. Pend., Kom. dan Pem. Huk. Isl., 9, 1N. Yuanita, M. A. Rini \& U. Heriawan. (2011). Coastal Engineering Practice

[39] R. D. Nasihien, D. A. R. Wulandari, A. Zacoeb,\& M. I. Setiawan. (2017). J. Darussalam: J. Pend., Kom. dan Pem. Huk. Isl., 9, 1

[40] S. A. Putra, B. Riyanto, A. Harsoyo \& A. I. Kistijantoro. (2015). TELKOMNIKA (Telecommunication Computing Electronics and Control) 13,3

[41] S. Exchange. (2013). Performance Summary. Jakarta: PT Pembangunan Jaya Ancol Tbk

[42] S. Ghavidelfar, A. Y. Shamseldin, \& B. W. Melville. (2017). J. of Environmental Planning and Management 60, no. 10

[43] S. J. Lee, D. Kim, K. R. Parrott, V. L. Giddings, \& S. R. Robinson. (2017). Housing and Society 44, no. 1-2: 4-21.

[44] S. Li, L. Wang \& K. L. Chang. (2017). Housing Studies

[45] S. M. Li \& Y. Liu. (2017). Cities 71

[46] S. Wetzstein. (2017). Urban Studies 54, 14

[47] Setiawan. (2003). Neutron, 3

[48] Setiawan. (2006). J. Neutron, 6, 2

[49] T. Economist. (2014). Investing Into Asia's Reform Investment Coordinating Board, Domestic Direct Investment Realization PMAQ4-2014. Jakarta, Indonesia

[50] T. F. King \& J. S. Feldman. (2017). Do People Have a Place in the Historic Environment? The Anderson Ferry and the national Mall. The Historic Environment: Policy \& Practice

[51] T. Huang, W. Gong \& G. Dai. (2011). Chinese Journal of Rock Mechanics and Engineering

[52] T. Okamoto, J. Yatsuhashi \& N. Mizutani. (2017). Proceedings of the 4th Multidisciplinary International Social Networks Conference on $\mathrm{ZZZ}$.

[53] UNCTAD United Nations Conference on Trade and Development (2013). World Investment Prospects Survey 2013-2015. United Nations: New York and Geneva

[54] Y. Lai, X. Zheng, L. H. T. Choy \& J. Wang. (2017). Land Use Policy 68

[55] Y. Fan, J. Wu \& Z. Yang. (2017). Regional Science and Urban Economics 67

[56] Y. Pribadi. (2015). Studia Islamika 22, 2

[57] Moeljarto, T., dkk. (2001). Birokrasi dalain Polernik, Pustaka Pelajar bekerjasama dengan Pusat Studi Kewilayahan Universitas Muhammadiyah Malang. 